\title{
Erratum: Angular dependence of spin-orbit spin-transfer torques [Phys. Rev. B 91, 144401 (2015)]
}

Ki-Seung Lee, Dongwook Go, Aurélien Manchon, Paul M. Haney, M. D. Stiles, Hyun-Woo Lee, and Kyung-Jin Lee (Received 3 May 2016; published 16 May 2016)

DOI: 10.1103/PhysRevB.93.179901

The sign convention is not consistent in some parts of the paper. In order to achieve the consistency, the following two equations should be modified. Equation (1) should be altered as follows,

$$
\mathbf{T}=\tau_{\mathrm{f}} \hat{\mathbf{M}} \times \hat{\mathbf{y}}-\tau_{\mathrm{d}} \hat{\mathbf{M}} \times(\hat{\mathbf{M}} \times \hat{\mathbf{y}})
$$

where the sign of the dampinglike spin-orbit torque (second term on the right-hand side) has been altered from + to - . Also, Eq. (8) should be altered as follows,

$$
\mathbf{s}_{(1)}^{ \pm}=\int \frac{d k^{2}}{(2 \pi)^{2}}\left[f_{ \pm}\left(\mathbf{k}+\frac{e E \tau}{\hbar} \hat{\mathbf{x}}\right)-f_{ \pm}(\mathbf{k})\right] \mathbf{s}_{\mathbf{k}(1)}^{ \pm},
$$

where the sign in front of the momentum shift $e E \tau / \hbar$ due to the electric field has been altered from - to + . After these two modifications, the sign convention becomes consistent in the paper.

The signs of the resulting $\tau_{\mathrm{f}}$ and $\tau_{\mathrm{d}}$ depend on the signs of $\alpha_{\mathrm{R}}$ and $E$. Regardless of the latter signs, however, the sign of the product $\tau_{\mathrm{f}} \tau_{\mathrm{d}}$ is positive (Figs. 2 and 3). This prediction is consistent with predictions of other theoretical works [1-3] on the Rashba spin-orbit coupling effect. To verify this point, it is convenient to check instead the following vector inequality,

$$
\left(\hat{\mathbf{M}} \times \mathbf{T}_{\mathrm{f}}\right) \cdot \mathbf{T}_{\mathrm{d}}<0,
$$

which is independent of various choices of sign conventions used in different works. Here, $\mathbf{T}_{\mathrm{f}}\left(=\tau_{\mathrm{f}} \hat{\mathbf{M}} \times \hat{\mathbf{y}}\right)$ and $\mathbf{T}_{\mathrm{d}}\left[=-\tau_{\mathrm{d}} \hat{\mathbf{M}} \times\right.$ $(\hat{\mathbf{M}} \times \hat{\mathbf{y}})]$ are the fieldlike and dampinglike spin-orbit torques, respectively.

[1] H. Kurebayashi, J. Sinova, D. Fang, A. C. Irvine, T. D. Skinner, J. Wunderlich, V. Novák, R. P. Campion, B. L. Gallagher, E. K. Vehstedt, L. P. Zarobo, K. Vyborny, A. J. Ferguson, and T. Jungwirth, Nat. Nanotechnol. 9, 211 (2014).

[2] H. Li, H. Gao, L. P. Zârbo, K. Výborný, X. Wang, I. Garate, F. Doğan, A. Čejchan, J. Sinova, T. Jungwirth, and A. Manchon, Phys. Rev. B 91, 134402 (2015).

[3] P. M. Haney, H.-W. Lee, K.-J. Lee, A. Manchon, and M. D. Stiles, Phys. Rev. B 87, 174411 (2013). 